https://revistas.ucr.ac.cr/index.php/ingenieria/index www.ucr.ac.cr / ISSN: 2215-2652
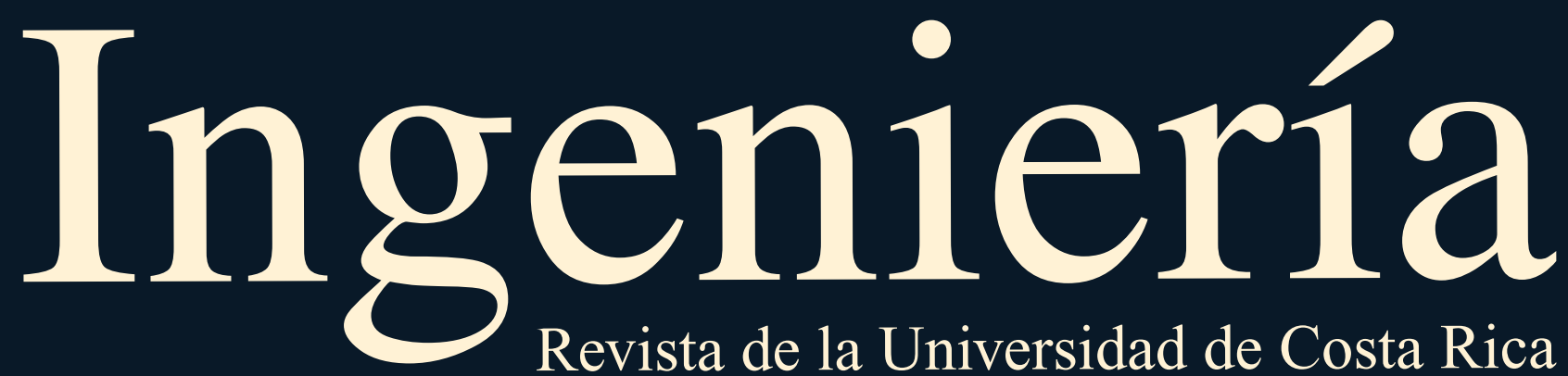

Revista de la Universidad de Costa Rica ENERO/JUNIO 2022 - VOLUMEN 32 (1)
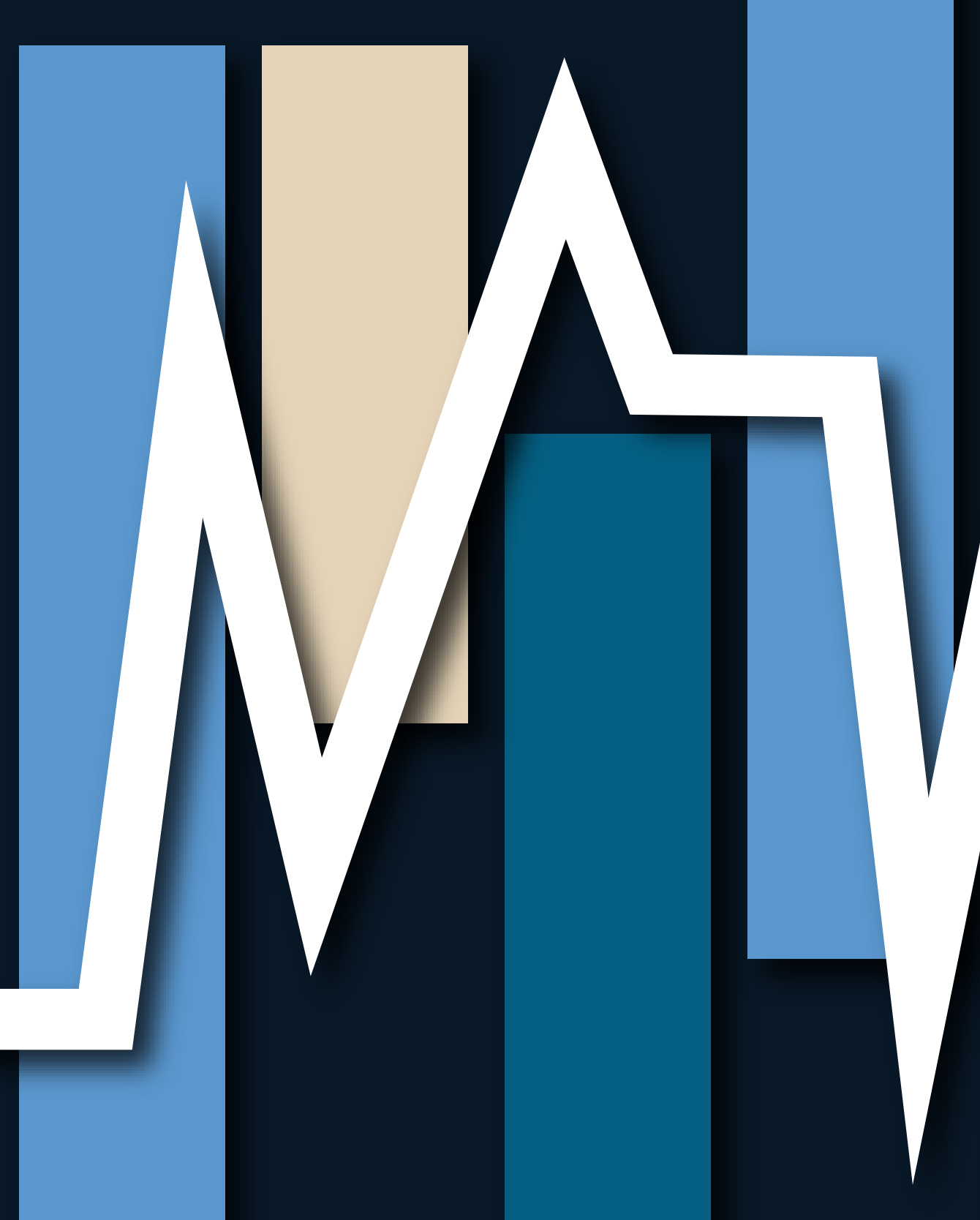


\title{
Comparativa multi e hiperespectral del pasto Cynodon nlemfuensis bajo condiciones tropicales y de pastoreo con ganado lechero
}

\section{Hyper and multi-spectral comparison of Cynodon nlemfuensis pasture under tropical and grazing conditions with dairy cattle}

\author{
Alberto Francisco Coto Fonseca \\ Estudiante, Posgrado en Ingeniería de Biosistemas \\ Universidad de Costa Rica, San José, Costa Rica \\ Email:alberto.cotofonseca@ucr.ac.cr \\ ORCID: 0000-0003-2732-6943 \\ Alejandra María Rojas González. \\ Profesora Asociada e Investigadora \\ Universidad de Costa Rica, San José, Costa Rica \\ Email: alejandra.rojasgonzalez@ucr.ac.cr \\ ORCID: 0000-0001-7984-7789
}

Recibido: 8 de marzo 2021

Aceptado: 28 de junio 2021

\begin{abstract}
Resumen
La información espectral ha sido utilizada ampliamente en el estudio de las condiciones nutricionales y en el desarrollo de diversos cultivos empleados en el ámbito agrícola, pero existe un vacío de investigación en especies forrajeras para condiciones tropicales. El presente estudio contempló la comparación de información multi e hiperespectral correspondientes al cultivo de pasto Estrella Africana (Cynodon nlemfuensis) dedicado a la alimentación de ganadería lechera usando espectroscopia de campo e información satelital de Sentinel-2. Se determinó que existe una heterogeneidad de la firma espectral del cultivo, debido al patrón aleatorio de alimentación por parte del ganado y la variación de las condiciones ambientales. Se generaron zonas con distintas alturas del cultivo, influyendo directamente en los valores de reflectancia, índice de área foliar e índices de vegetación. Se compararon los índices espectrales calculados con información de campo y satelital, obteniéndose valores de $\mathrm{R}^{2}$ de 0,725 para el caso del NDVI y de 0,446 para el SAVI. La presente investigación es de gran relevancia ya que sienta la línea base del uso de información espectral para el estudio de pastos dedicados a la alimentación de ganado lechero a partir de sensores remotos y espectrorradiometría de campo.
\end{abstract}

Palabras clave:

Espectrorradiometría, firma espectral, NDVI, SAVI, SENTINEL 2-MS. 


\begin{abstract}
Spectral information has been widely applied to study the growth and nutritional conditions of different crops used in the agricultural field; however, there is a research gap regarding forage crops in tropical conditions. This study compared the multi and hyperspectral information of the African star pasture crop (Cynodon nlemfuensis) for dairy cattle feeding using field spectroscopy and satellite information of Sentinel-2. This study determined spectral signature heterogeneity of this crop due to the randomness of the feeding pattern of the cattle and the continuous change of the environmental conditions. Different crop heights in the sampling areas affected the reflectance values, leaf area index and vegetation indices directly. For the NDVI and SAVI, $\mathrm{R}^{2}$ values of 0,725 and 0,446 were achieved for spectral indices between field and satellite data. This research is relevant because it lays the baseline for the use of spectral information regarding the analysis of tropical pastures employed in dairy cattle feeding using remote sensing and a field spectroradiometer.
\end{abstract}

\title{
Keywords:
}

NDVI, SAVI, SENTINEL 2-MS, spectral signature, spectroradiometry. 


\section{INTRODUCCIÓN}

El uso de sensores remotos ha sido un tema de gran relevancia para el estudio de la vegetación permitiendo analizar de manera no destructiva la fenología y su monitoreo, la estimación del rendimiento y materia orgánica [1], [2], así como el requerimiento hídrico [3].

Otro uso de los sensores remotos es el cálculo de la evapotranspiración real de los cultivos. Para dicho cálculo se utilizan modelos basados en balances de energía e índices espectrales de información satelital, generalmente de Landsat 8 [4], [5]. Dentro de estos índices, se encuentran el índice de vegetación de diferencia normalizada (NDVI por siglas en inglés) y el índice de vegetación ajustado al suelo (SAVI por siglas en inglés). Estos índices han sido utilizados en aplicaciones tales como la generación de modelos para el estudio de los requerimientos hídricos de los cultivos, sirviendo así como una guía para la toma de decisiones en relación a dicha temática [6], [7]. Además, estos modelos han llegado a generar resultados muy cercanos al estado real de los cultivos [8] así como la estimación de los coeficientes basales [9].

Dentro de los principales satélites utilizados para obtener información multiespectral se encuentran Sentinel-2 con una resolución espacial de 10 m o 20 m (dependiendo de la banda) y temporal de 5 días [10] y Landsat 8 con resoluciones de $30 \mathrm{~m}$ y 16 días respectivamente [11]. De ambos satélites, Sentinel-2 es el más reciente y desde su lanzamiento ha sido utilizado en estudios relacionados con la cobertura vegetal. Dentro de sus aplicaciones, se encuentra la determinación de parámetros biofísicos asociados a cultivos agrícolas tales como el índice de área foliar, el contenido de clorofila del dosel y de la hoja [12] y la estimación de biomasa en pastizales a partir de índices de vegetación tales como el NDVI [13].

Al ser información generada por tecnología satelital, es necesaria su validación mediante datos obtenidos en el campo, de manera que los resultados sean aptos para ser utilizados como referencia para la toma de decisiones en relación con el mantenimiento de los cultivos. Una manera de realizar dicha validación es mediante el uso de espectrorradiometría de campo.

Actualmente, la espectrorradiometría de campo tiene una gran aplicabilidad en varios campos del conocimiento. Dentro de sus aplicaciones, se encuentra el estudio de propiedades del suelo [14]-[16]; identificación de la presencia de metales pesados en suelos utilizados en actividades tales como la minería [17], o bien como una manera de reclamar terrenos que están siendo utilizados en dicha actividad [18], de forma tal que sirven como una guía y monitoreo del adecuado uso del suelo bajo el marco de un desarrollo sostenible con el ambiente.

Dentro del sector agrícola, la espectrorradiometría ha sido utilizada para analizar temas como la identificación de nutrientes en los suelos dedicados a esta actividad [19] y el estudio del estado de los cultivos, con el fin de reconocer patrones que sirvan para la identificación de ciertas enfermedades [20].

Considerando lo anterior, y que la mayoría de los estudios se direccionan a cultivos dedicados a la alimentación humana, como por ejemplo el arroz [21], es importante orientar estos análisis a cultivos dedicados a otras actividades productivas. En esta línea de investigación se han llevado a 
cabo estudios relacionadas con la producción de pastos. Dentro de estos, se encuentra el realizado por Pullanagari et al. [22], quienes evaluaron las diferencias espectrales entre diversos tipos de pastos y generaron relaciones entre información de reflectancia y parámetros de calidad química como proteína cruda, cenizas, lignina y energía metabólica.

En Costa Rica, la producción de forrajes es de gran relevancia para el sector productivo ganadero al ser fuente principal de alimento para el ganado. Dentro de los principales pastos que se producen, está la Estrella Africana (Cynodon nlemfuensis). Esta es una especie que, por sus características de adaptación, nutrición y producción en un amplio rango de ambientes y tipos de suelos, se utiliza en gran medida en los sistemas ganaderos dedicados a la producción lechera en el país [23].

En vista de la importancia que tiene la producción de pasto, se identificó que a nivel nacional no se han realizado estudios asociados al uso de tecnologías de teledetección que analicen el comportamiento espectral de especies de pastos tropicales bajo pastoreo de ganado lechero. Adicionalmente, existe una necesidad de mejorar la estimación de variables asociadas a la producción de forrajes en los sistemas productivos lecheros (p.ej biomasa).

Considerando lo anterior y el gran potencial de los sensores remotos para el análisis de la vegetación, el surgimiento de mejores tecnologías satelitales y la potencial diferencia que puede haber entre los datos espectrales de satélite y de espectrorradiometría de campo destinados a pastos tropicales, es que la presente investigación tuvo como objetivo principal comparar la información hiper y multi-espectral asociada al cultivo de pasto Estrella Africana bajo condiciones de pastoreo de ganado lechero, mediante espectrorradiometría de campo e información satelital a partir de Sentinel-2.

\section{MATERIALES Y MÉTODOS}

\subsection{Sitio de muestreo}

El estudio se llevó a cabo de octubre a diciembre (período lluvioso e inicio de época seca) del año 2019 en la Estación Experimental de Ganado Lechero Alfredo Volio Mata (EEGLVM) de la Universidad de Costa Rica. La estación se localiza en Ochomogo en el distrito de San Rafael, cantón de la Unión de la provincia de Cartago, latitud de $9^{\circ} 54^{\prime} 42,11^{\prime \prime} \mathrm{N}$, longitud 8357'19,12”O, a una altura de 1568 m.s.n.m, donde los suelos son, en su mayoría andisoles [24]. Esta finca cuenta con ganado lechero de raza Jersey y se cultivan diferentes pastos forrajeros, entre los más importantes pasto Estrella Africana, Taiwán y Botón de Oro.

Del total de parcelas que tiene la EEGLAVM, veinte y ocho son dedicadas al pastoreo de ganado lechero con pasto Estrella Africana (Cynodon nlemfuensis). En estas parcelas se trabaja con un sistema de apartos, donde cada potrero es subdividido en cuatro secciones. En cada uno de ellos el ganado ingresa dos veces al día para alimentarse y cada potrero tiene una duración aproximada de cuatro días en ser pastoreado completamente. Debido a la rotación del ganado a lo largo de las parcelas, cada una de ellas inicia nuevamente el ciclo de pastoreo dentro de aproximadamente 25 días. 
De las veinte y ocho parcelas mencionadas, la toma de datos se llevó a cabo dentro de dos parcelas en tres áreas muestrales (denominadas A, B y C). Las áreas muestrales consisten en clústeres de 3 x 3 pixeles [25] de $10 \mathrm{~m}$ de resolución, tal y como se muestra en la Fig. 1. Dichas zonas de muestreo se identificaron y localizaron en el campo mediante el uso de estacas en los cuatro vértices de cada área muestral y por medio de GPS marca Garmin modelo Oregon 550.

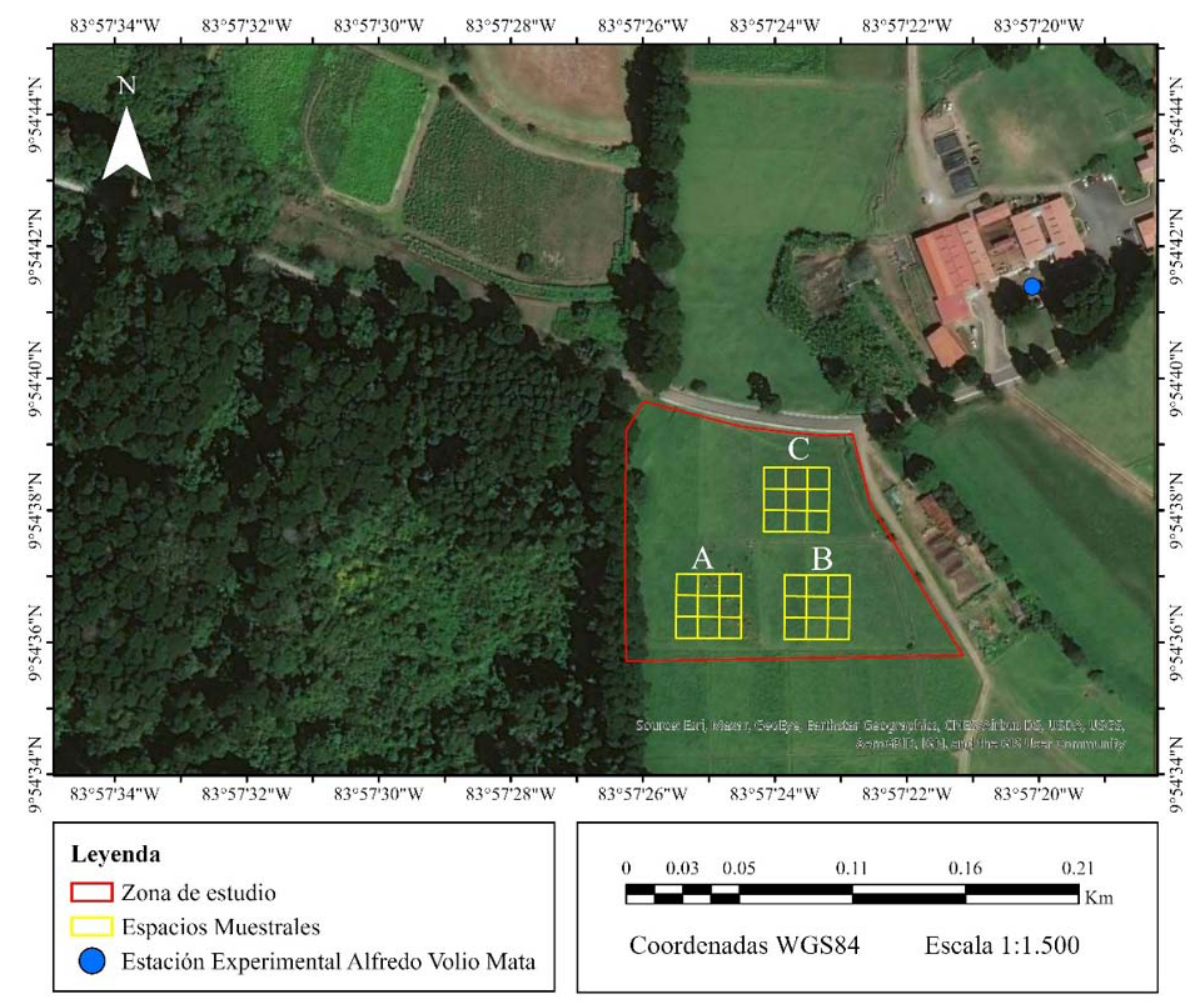

Fig. 1. Ubicación de las zonas de muestreo (clústeres) de pasto Estrella Africana en la EEGLVM.

\subsection{Mediciones}

Para cada espacio muestral, se recolectó, en campo, información correspondiente al índice de área foliar (IAF), firma espectral del cultivo y la altura del pasto. El IAF se midió mediante un analizador de dosel LAI 2200C marca LI-COR. Para dicho índice, se tomaron dos mediciones sobre el pasto y cinco valores debajo de la cobertura en un trayecto diagonal para un total de tres repeticiones por píxel de $10 \mathrm{~m}$.

La información hiperespectral se obtuvo mediante un espectrorradiómetro ASD FieldSpec 4 Wide Resolution, con resolución espectral de $3 \mathrm{~nm}$ para el rango de medición de longitud de onda entre 350 a $1000 \mathrm{~nm}$ y de $10 \mathrm{~nm}$ para la región de 1000 a $2500 \mathrm{~nm}$. El levantamiento de los datos espectrales se realizó a una altura aproximada de $1 \mathrm{~m}$ desde el nivel de suelo, para un diámetro de cobertura óptica aproximada de $44 \mathrm{~cm}$. Las mediciones se llevaron a cabo entre las 8 am y $1 \mathrm{pm}$. Se consideró dicha ventana de tiempo para la toma de datos, de manera que se tuvieran condiciones ambientales adecuadas para no alterar la información espectral a recolectar [26] y se coincidiera con el paso del satélite (alrededor de las 10:10 am). 
La configuración del espectrorradiómetro utilizada fue de 100 mediciones de corriente oscura y 30 mediciones de blancos de referencia. Para cada pixel, se utilizó un promedio de 20 espectros por medición [27], se realizó un muestreo en cruz por píxel [28], donde se realizaron cuatro repeticiones en cada punto para un total de 16 firmas por píxel y así capturar la variabilidad presente.

Se realizó la optimización del equipo y la toma del blanco de referencia con un Lambertian White Reference (spectralon 5 x $5 \mathrm{~cm}$ ) cada 10 minutos o menos, dependiendo de la variación de la iluminación generada por las condiciones ambientales.

En el caso de la información multiespectral, se utilizaron imágenes del satélite Sentinel-2 nivel 2A (con corrección atmosférica), las cuales tienen una resolución temporal de 5 días y espacial de $10 \mathrm{~m}$ (bandas rojo, azul, verde y NIR). Estas se utilizaron para el cálculo de los índices de vegetación NDVI y SAVI. Se recolectaron todas las imágenes disponibles durante el período de estudio (http://www.sentinel.esa.int/web/sentinel-data-access), de las cuales, finalmente, solo dos fueron utilizadas, ya que las demás presentaban nubosidad sobre la zona de estudio.

\subsection{Post-procesamiento de datos y cálculo de índices espectrales}

La información hiperespectral fue procesada con el software ViewSpec Pro Version 6.2 [29]. Un control de calidad inicial de las firmas hiperespectrales fue realizado de manera visual para identificar problemas o cambios repentinos en la intensidad de luz de los espectros que aumenten el ruido de las mediciones.

A partir de los datos hiperespectrales obtenidos, se calculó la firma espectral promedio para el pixel, usando los 16 espectros recolectados y se generó la firma promedio para cada zona muestral (9 pixeles). Adicionalmente, se promedió la información de reflectancia de campo con base en los rangos de referencia de las respuestas espectrales del satelite Sentinel-2 de la banda 4 correspondiente al rojo (645 nm a $685 \mathrm{~nm}$ ) y la banda 8 que corresponde al infrarojo cercano (NIR) (774 $\mathrm{nm}$ a $907 \mathrm{~nm}$ ) [30]. Lo anterior para calcular los índices de vegetación y realizar una comparación pixel a pixel con los índices generados a partir de la información satelital y de campo. Se utilizó la Plataforma de Aplicación de Sentinel (SNAP) de la Agencia Europea Espacial (ESA) para obtener los valores espectrales de cada uno de los pixeles en estudio, mediante la extracción de los valores de reflectancia de cada una de las bandas que componen las imágenes satelitales de Sentinel-2, con los cuales se generó una firma promedio para cada sitio muestral y su posterior comparación con la información de campo.

\section{CUADRO I}

FÓRMULAS DE LOS ÍNDICES DE VEGETACIÓN

\begin{tabular}{cccc}
\hline Índice & \multicolumn{1}{c}{ Descripción } & Fórmula & Fuente \\
\hline NDVI & $\begin{array}{c}\text { Índice de Vegetación de } \\
\text { Diferencia Normalizada } \\
\text { Índice de Vegetación } \\
\text { Ajustado al Suelo }\end{array}$ & $\begin{array}{c}\frac{N I R-\text { Rojo }}{N I R+\text { Rojo }} \\
\frac{(1+L)(N I R-R o j o) *}{L+(N I R+R o j o)}\end{array}$ & {$[32]$} \\
\hline
\end{tabular}

*Donde $L$ es una constante para reducir el sesgo, debido a efectos del fondo del suelo, asignándose un valor de 0,1 [34]. 
Se calcularon dos índices de vegetación: el índice de vegetación de diferencia normalizada (NDVI) y el índice de vegetación ajustado al suelo (SAVI). Dichos índices fueron generados en el software QGIS v 3.10.2 [31], utilizando las ecuaciones presentadas en el CUADRO I y los rangos de reflectancia indicados anteriormente.

\subsection{Análisis estadístico}

Se utilizó la prueba de Shapiro-Wilk para determinar la normalidad de los datos de índice de área foliar e índices espectrales (satelital y espectral). Posteriormente, se analizó si existen diferencias significativas entre los sitios de muestreo para las variables mencionadas. Esto se comprobó mediante pruebas de hipótesis nula a partir de análisis de varianza (ANOVA) (paramétrico con más de dos poblaciones) o Kruskal Wallis (equivalente del ANOVA para casos no paramétricos). Para el caso de dos poblaciones, se utilizó la prueba T-Student (paramétrico) o Mann-Whitney (no paramétrico) dependiendo de cada caso. De encontrarse dichas diferencias con el ANOVA y Krustal-Wallis, se implementó la prueba post hoc de Tukey y Dunn's, respectivamente. Para lo anterior, se usó el software Past v.3.25 [35].

\subsection{Métricas de rendimiento}

Para evaluar los datos obtenidos entre la información generada en campo y la satelital, se calculó el coeficiente de determinación $\left(\mathrm{R}^{2}\right)$, la raíz del error cuadrático medio (RECM) y el error absoluto medio (EAM).

\section{RESULTADOS Y DISCUSIÓN}

En el periodo de tiempo que se condujo el estudio, fue posible realizar el levantamiento de información hiperespectral en 8 fechas coincidentes con el paso del satélite Sentinel-2 por Costa Rica. Adicionalmente, se recolectó información de la altura del cultivo ( 6 fechas) y el IAF (4 fechas) para cada parcela de medición.

En la Fig. 2, se presenta el comportamiento temporal promedio de las firmas hiperespectrales para el sitio muestral B como ejemplo, donde se observa un patrón definido del 13 de octubre al 12 de diciembre. Conforme se desarrolla el cultivo, los valores de reflectancia en la región del infrarrojo cercano (NIR) aumentan; sin embargo, el 2 de noviembre la firma hiperespectral se reduce, coincidiendo con la reducción de la altura del pasto, producto de la alimentación del ganado lechero seis días antes de la medición. Posteriormente, hay un aumento de la reflectancia a partir del 12 de noviembre, al comenzar nuevamente el crecimiento del pasto hasta llegar a un valor máximo el 12 de diciembre del 2019.

Al pasar el tiempo, los valores de reflectancia en el rango infrarrojo cercano aumentan paulatinamente hasta el 23 de octubre. El 2 de noviembre, los valores de reflectancia del pasto presentan una fuerte disminución, debido a la introducción del ganado seis días antes para su pastoreo, luego vuelven a aumentar según el pasto va creciendo, hasta llegar a su punto máximo el 12 de diciembre. 


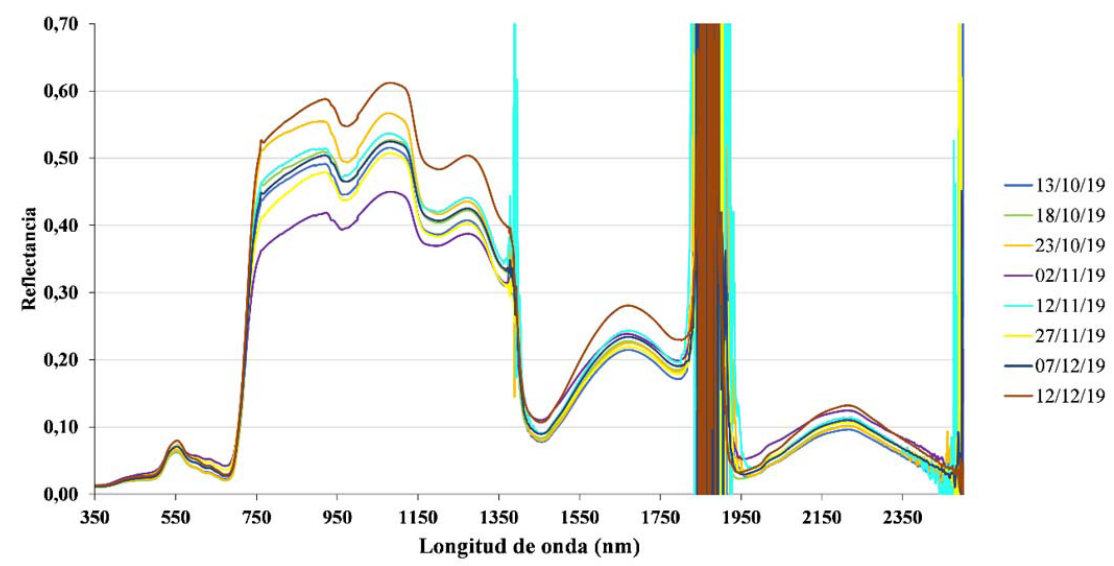

Fig. 2. Comparación temporal de firmas espectrales del sitio muestral B para el período de estudio.

La región del infrarrojo cercano (700 nm a $1350 \mathrm{~nm}$, aproximadamente) es de gran relevancia, ya que está relacionado con la estructura celular de las plantas, de manera que en muchos casos es utilizada para la detección e identificación de enfermedades presentes en las hojas, tal es el caso del reconocimiento de patógenos fúngicos [36], [37], mientras que el rango de los $350 \mathrm{~nm}$ a 780 $\mathrm{nm}$ está relacionado con la absorción de clorofila. Respecto a esta última región, estudios como el de Croft et al. [38] analizan el potencial del uso de información multiespectral de plataformas como Landsat 8 para determinar el contenido de clorofila de la vegetación en cultivos agrícolas, tales como maíz y trigo, con el fin de mejorar la toma de decisiones respecto a aspectos nutricionales de los cultivos.

Conforme crece el pasto, el mismo absorbe una mayor cantidad de luz en el espectro azul (450 $\mathrm{nm})$ y rojo $(670 \mathrm{~nm})$, debido al aumento en su contenido de clorofila para así potenciar el proceso fotosintético y, por ende, reflejar una mayor cantidad de energía en el rango NIR. Por esta razón, las plantas sanas presentan mayores valores de reflectancia en este rango que aquellas que se encuentran enfermas, por lo que puede ser utilizado como un indicador del estado de salud y crecimiento del pasto.

Se presenta en la Fig. 2. una región de ruido en los rangos de $1350 \mathrm{~nm}$ a los $1400 \mathrm{~nm}$ y de $1800 \mathrm{~nm}$ a los $1950 \mathrm{~nm}$, las cuales están relacionados a efectos de humedad en el ambiente en referencia de los blancos obtenidos mediante el espectralon [39]. Esta condición siempre se presenta a la hora de realizar mediciones en campo, debido a las condiciones ambientales imperantes de la zona y la única forma de disminuir dicho ruido es mediante el uso de accesorios con luz artificial o bien realizando las mediciones en el laboratorio, las cuales no son contempladas en este estudio.

Tal y como se muestra en los últimos días (noviembre 27, diciembre 7 y 12) de la Fig. 2, se observó el mismo comportamiento de aumento mencionado de la firma hiperespectral; sin embargo, la firma del 7 de diciembre es la que presenta los valores de reflectancia más altos, aun presentando una altura de cultivo menor a la de los días anteriores. Es importante mencionar que no todos los días en los que se realizaron las mediciones (octubre y noviembre) estuvieron completamente 
despejados al realizarse el estudio en época lluviosa, lo cual pudo influir directamente sobre las firmas espectrales obtenidas en campo.

La Fig. 3 presenta el comportamiento del crecimiento del pasto Estrella Africana durante el período de estudio en las parcelas A, B y C. Los resultados concuerdan con lo observado en la Fig. 2, donde en un inicio se presenta un crecimiento $y$, posteriormente, la altura del pasto decae al ingresar el ganado para su pastoreo.

De las tres zonas, la $\mathrm{C}$ es la que presenta, en el período de análisis, la mayor altura del cultivo. La altura máxima promedio fue de $58 \mathrm{~cm}$ para la parcela $\mathrm{C}$, registrada el 23 de octubre, (Fig. 3) mientras que la parcela A y B presentaron alturas de $47 \mathrm{~cm}$ y $46 \mathrm{~cm}$, respectivamente. Cabe destacar que la parcela $\mathrm{C}$ limita con un canal abierto en tierra, el cual recibe parte de las aguas tratadas provenientes de la lechería de la EEGLVM. Estos aportes potenciales le proporcionan nutrientes por infiltración a la parcela $\mathrm{C}$ que le permiten un mayor crecimiento.

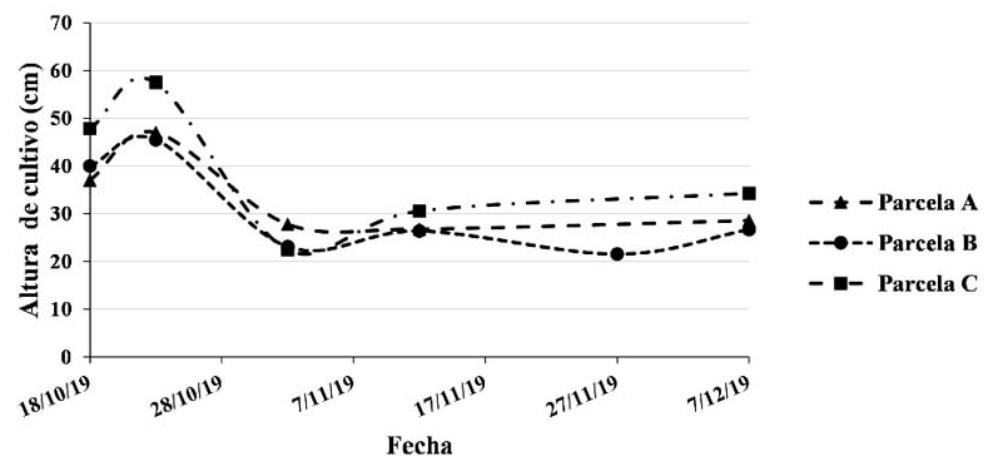

Fig. 3. Altura del cultivo de pasto Estrella Africana a lo largo del período de estudio.

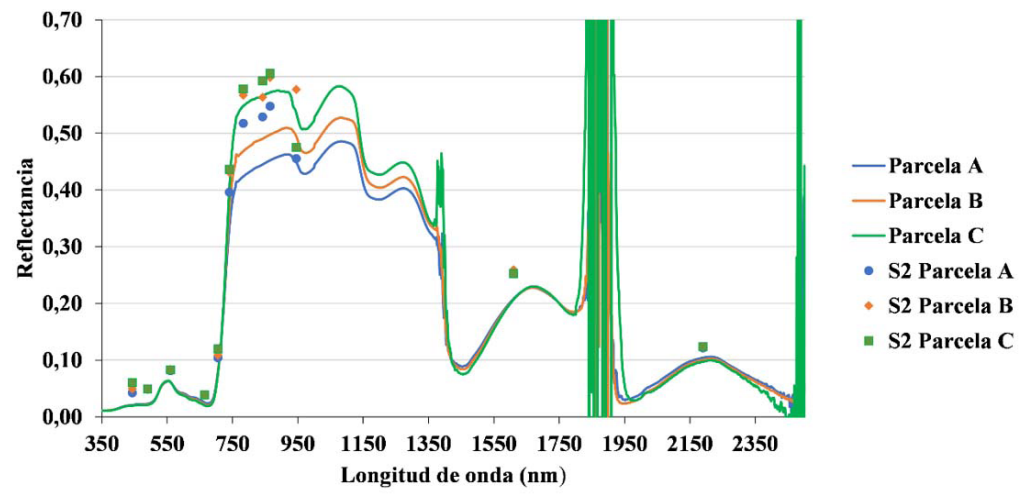

Fig. 4. Comparación de información espectral de las tres zonas de muestreo para datos de campo (espectrometría, líneas continuas) y satelitales (Sentinel 2, puntuales) para el 18 de octubre de 2019.

En la Fig. 4, se detallan las firmas espectrales promedio para los datos de campo medidas con el espectrorradiómetro (líneas continuas) y del satélite Sentinel A (formas puntuales en la gráfica) para las tres zonas de muestreo estudiadas para el 18 de octubre de 2019, como ejemplo. Se aprecia una diferencia en los espectros, la cual se atribuye al estado de desarrollo del pasto, donde las zonas A, B y C presentan alturas promedio de $37 \mathrm{~cm}, 40 \mathrm{~cm}$ y $48 \mathrm{~cm}$, respectivamente. En el rango espectral 
entre los $1450 \mathrm{~nm}$ a $1760 \mathrm{~nm}$ y de $\operatorname{los} 1950 \mathrm{~nm}$ a $\operatorname{los} 2300 \mathrm{~nm}$, las variaciones en reflectancia entre las parcelas son leves. Todas las áreas de muestreo presentaron heterogeneidad correspondiente al estado del cultivo (Fig. 4), debido a que, al ser un área dedicada al pastoreo de ganado lechero, se depende de la programación establecida para la alimentación del ganado.

Respecto a la información de reflectancia satelital, tal y como se observa en la Fig. 4, se nota el mismo comportamiento respecto a los datos de reflectancia obtenidos en campos. Sin embargo, se identificó que la reflectancia de las bandas que componen la imagen de Sentinel-2 sobreestiman la obtenida a partir de espectrorradiometría de campo. Es en el rango NIR donde se denota un mayor distanciamiento entre los datos hiper y multiespectrales. Es importante recalcar que, al ser un píxel de 10 x 10 m, no se abarca toda la variabilidad inherente al campo, algo que sí se obtiene a partir de información hiperestral mediante el uso de espectrorradiometría de campo.

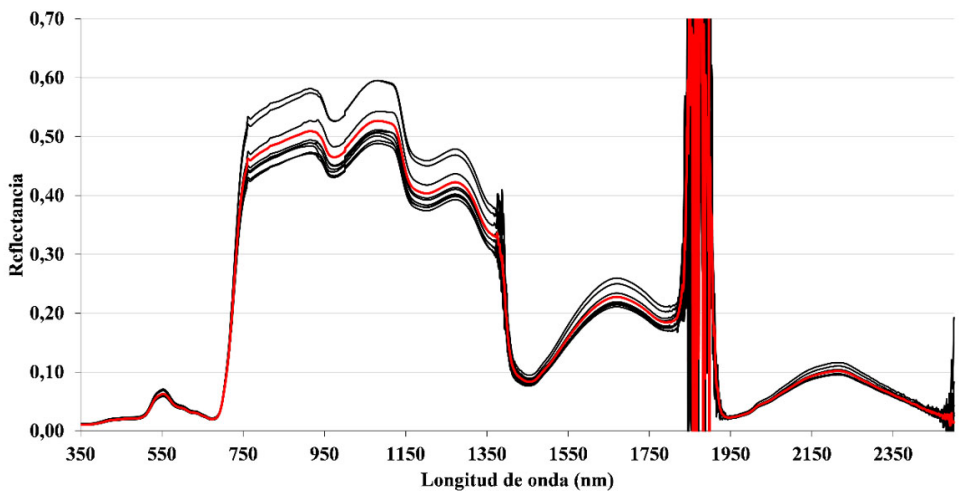

Fig. 5. Comparación espacial de firmas espectrales promedio por píxel a lo interno del sitio muestral B para el 18 de octubre de 2019. Firma de color rojo corresponde al promedio muestral

Se debe de tomar en cuenta que los patrones de pastoreo del ganado en campo son completamente aleatorios y generan una degradación irregular en el pasto, alterando los patrones de altura del pasto, compactación de suelos e, inclusive, dejando áreas descubiertas o suelo expuesto. Estas variaciones espaciales, dentro de un mismo espacio muestral, se evidencian en la Fig. 5, donde se presenta el comportamiento de las firmas espectrales promedio por píxel dentro de la zona de muestreo B. Al igual que en el caso anterior, difiere en su mayoría en el rango del infrarrojo cercano, con variaciones leves entre los $1450 \mathrm{~nm}$ a los $1760 \mathrm{~nm}$ y de los $1950 \mathrm{~nm}$ a los $2300 \mathrm{~nm}$. Considerando lo anterior, cada zona de muestreo presenta variabilidad interna en las firmas hiperespectrales que corresponden a los pixeles que la componen, tal y como se muestra en la Fig. 5. En esta se aprecia que, dentro del área de estudio, el pastoreo tiene un efecto directo sobre la dinámica del sistema del cultivo de pasto estrella, influyendo en el desarrollo de este y evitando que se tenga uniformidad.

Respecto a las mediciones del índice de área foliar realizadas en campo, existen cambios temporales (Fig. 6 (a)) y espaciales producto de la actividad de pastoreo del ganado en las parcelas (Fig. 6 (b)). El IAF evaluado con el analizador de dosel 2200C en cuatro fechas de medición están acorde a los cambios registrados del 13 al 23 de octubre, donde existe un comportamiento creciente, acorde con el desarrollo del cultivo y, por ende, una mayor cobertura del suelo. Se obtuvo un máximo 
IAF de 2,44 para la parcela A y 1,80 para las parcelas B y C. Posteriormente, el ganado ingresa a las parcelas y se mantienen los IAF en valores promedio de 1,34 en la segunda semana de noviembre y promedios de 1,68 para el 7 de diciembre.

El IAF permite reforzar lo encontrado en el comportamiento heterogéneo del desarrollo del cultivo bajo condiciones de pastoreo, ya que a lo largo del tiempo también presentó variabilidad entre las tres zonas de muestreo. Lo anterior porque el ganado no tiene un patrón definido a la hora de alimentarse en campo, por lo que se generan parches con distintas alturas de pasto (Fig. 6 (b)), sumado a la influencia ocasionada por la rotación de las parcelas en esta actividad. Esa situación fue comprobada de manera estadística, donde todas las fechas presentaron diferencias significativas entre algunos de los sitios de muestreo. Se determinó que efectivamente existen diferencias significativas $(\mathrm{p}<0,05)$ en el índice de área foliar. El 13 de octubre se obtuvieron diferencias para todas las áreas. El 18 de octubre, esas diferencias se presentaron entre las zonas A-B y A-C. En el caso del 12 de noviembre, los sitios de muestreo que presentaron diferencias corresponden al A-B y B-C y para la última fecha (7 de diciembre) se obtuvieron diferencias entre la zona A-B y B-C. Con relación a la variabilidad del IAF, es relevante mencionar que otro aspecto que influye en dicho resultado es la tasa de crecimiento del pasto. Lo anterior no solo por estar en distintas etapas de crecimiento, sino también por el efecto del pisoteo del ganado sobre el terreno [40] y la influencia de este sobre propiedades del suelo, tal como la densidad aparente [41], las cuales a su vez pueden influir en el crecimiento radicular del cultivo.
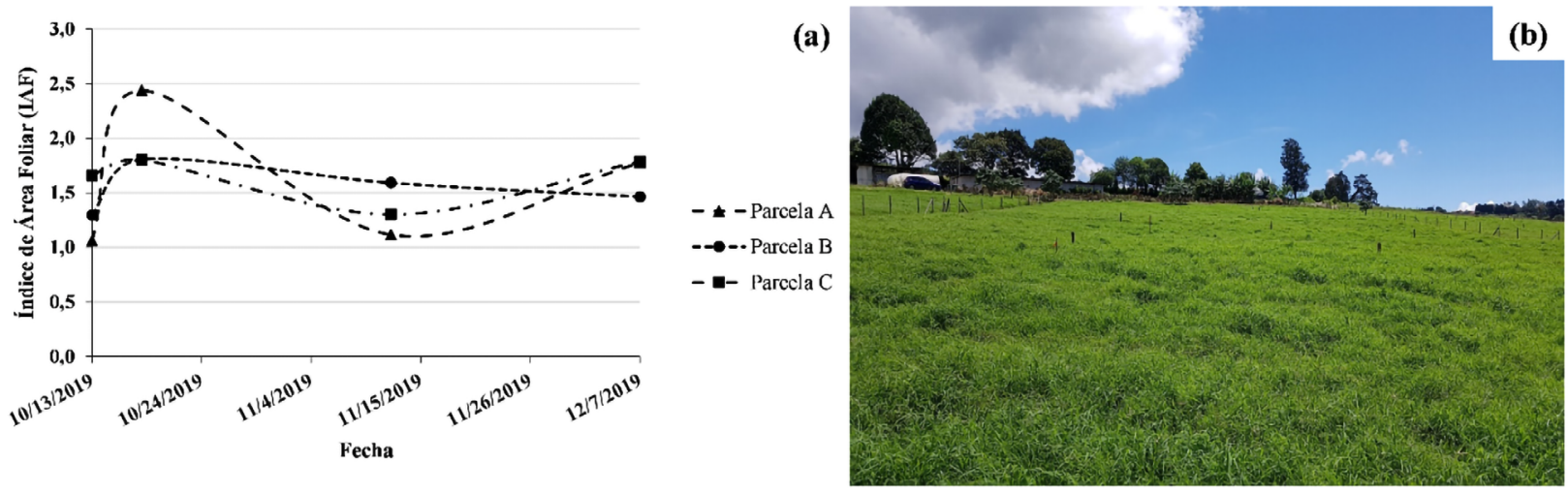

Fig. 6. Índice de Área Foliar a lo largo del tiempo (a) y condición del pasto para el 18 de octubre de 2019 (b).

En la Fig. 7, se presenta la comparación entre el índice de área foliar bajo estudio generado en campo con el predicho, calculado a partir de la herramienta SNAP de la ESA. Dicha figura demuestra que no se genera una correlación lineal entre ambos sets de datos. Tal y como se mencionó anteriormente, el IAF presenta una gran heterogeneidad, debido a la cobertura del pasto en campo; lo cual no queda reflejado, ya que la resolución espacial de la información satelital. En este sentido el IAF predicho con información satelital, sobrestima el obtenido en campo. Dicho comportamiento también ha sido obtenido en otros estudios, por ejemplo, el llevado a cabo en cultivos como maíz y girasol, donde el IAF generado con el SNAP también sobrestima los valores de campo [42]. 
Evaluando ambas informaciones se obtuvo un RECM de 3,49, un MAE de 3,42 y un $\mathrm{R}^{2}$ de 0,0082 (Fig. 7), reforzando la diferencia existente entre la información del IAF de campo y la generada con información satelital y la limitante existente en su estimación en pastos con pastoreo de ganado lechero con rotación de parcelas.

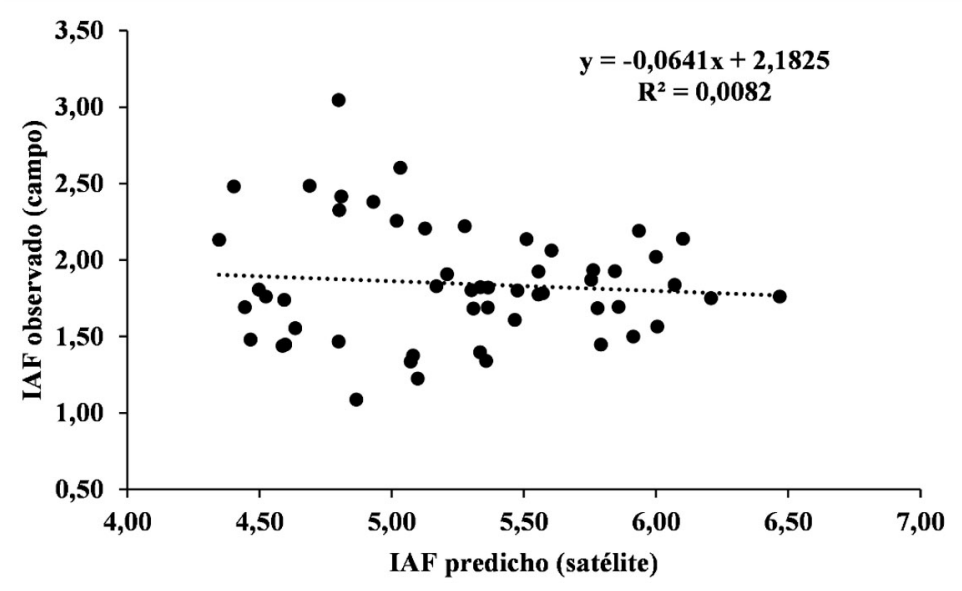

Fig. 7. Correlación de valores de IAF obtenidos en campo e información satelital

En el CUADRO II, se presentan los resultados de los índices espectrales (NDVI y SAVI) obtenidos tanto a nivel de campo como a nivel satelital para las dos imágenes analizadas, donde se obtuvieron diferencias significativas en ambas fechas para los índices calculados a partir de los datos de campo y de datos satelitales.

CUADRO II

ÍNDICES ESPECTRALES DE VEGETACIÓN DE LA ZONA DE ESTUDIO

\begin{tabular}{ccccc}
\hline Información & \multicolumn{2}{c}{$\mathbf{1 8 / 1 0 / 2 0 1 9}$} & \multicolumn{2}{c}{$\mathbf{7 / 1 2 / 2 0 1 9}$} \\
\hline & NDVI & SAVI & NDVI & SAVI \\
Campo & $0,910 \pm 0,018^{\mathrm{A}}$ & $0,839 \pm 0,031^{\mathrm{A}}$ & $0,886 \pm 0,021^{\mathrm{A}}$ & $0,819 \pm 0,023^{\mathrm{A}}$ \\
Satélite & $0,874 \pm 0,009^{\mathrm{B}}$ & $0,962 \pm 0,010^{\mathrm{B}}$ & $0,855 \pm 0,020^{\mathrm{B}}$ & $0,940 \pm 0,022^{\mathrm{B}}$ \\
\hline
\end{tabular}

Nota: letras distintas indican diferencias significativas.

Dichas diferencias se explican a partir de las correlaciones pertinentes entre los valores de campo y satélite para ambos índices de vegetación (Fig. 8 y Fig. 9). Se observa que existe una relación lineal donde los valores del coeficiente de determinación $\left(\mathrm{R}^{2}\right)$ son menores a 0,80. En esta temática, se han obtenido resultados similares, donde los $\mathrm{R}^{2}$ para pastos andinos fueron menores a 0,54, debido al efecto de saturación en las fibras ópticas del equipo durante la toma de datos, así como por la cantidad de mediciones realizadas [43]. En el presente estudio, el índice que presentó el mayor valor es el NDVI $\left(\mathrm{R}^{2}>0,725\right)$ 


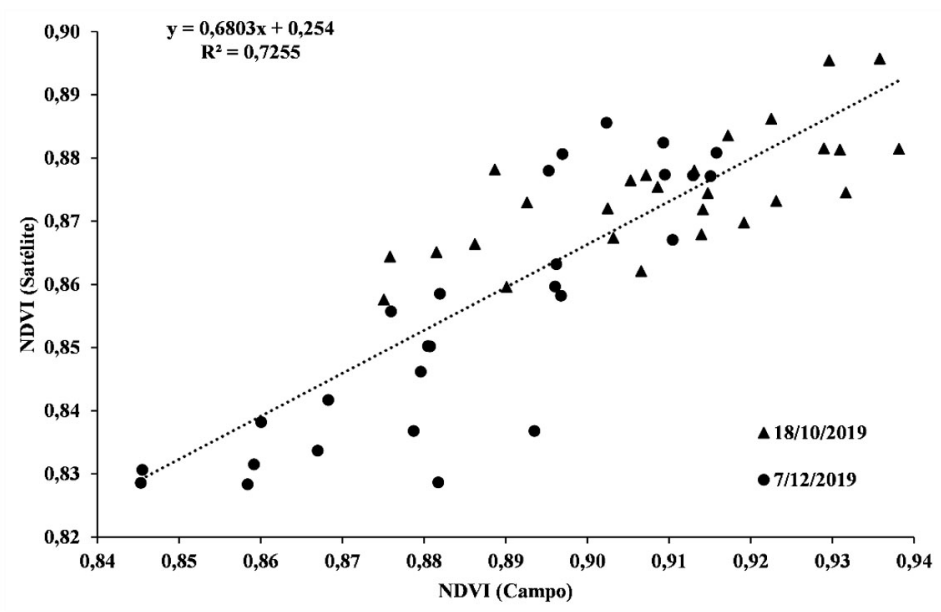

Fig. 8. Correlación de valores de NDVI obtenidos en campo e información satelital

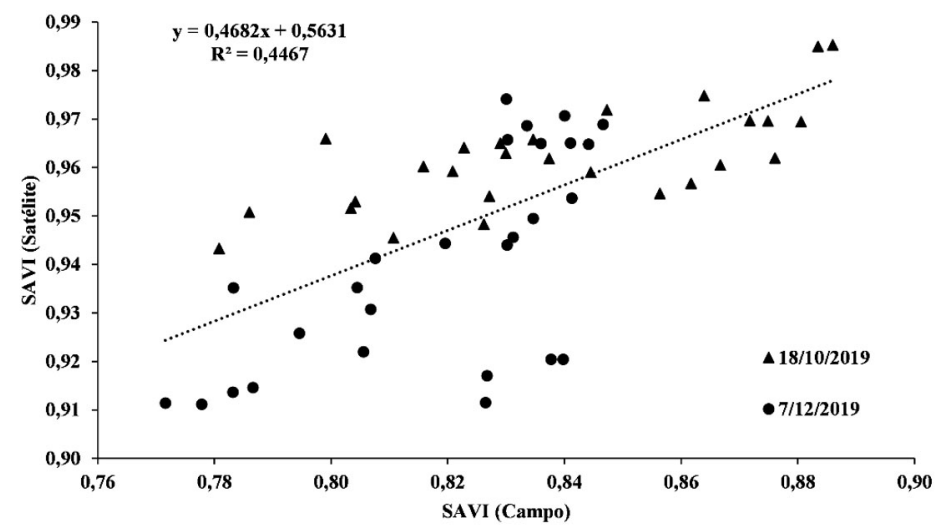

Fig. 9. Correlación de valores de SAVI obtenidos en campo e información satelital

El NDVI obtenido mediante la información espectral de campo (datos reales) y la información satelital presentaron un RECM de 0,035 y un EAM de 0,033. Se presume que la información satelital subestima la medición de campo.

En contraste, para el caso del SAVI, los valores satelitales sobrestiman las mediciones realizadas en campo. Para este índice de vegetación se obtuvo un RECM de 0,124 y un EAM de 0,122.

Aun teniendo este tipo de diferencias entre los valores de NDVI entre satélite y espectrorradiometría de campo, se obtuvo un valor aceptable de correlación de Pearson $(R>0,85)$. Es necesario indicar que los resultados obtenidos a partir de las firmas hiperespectrales están sujetas a las condiciones externas presentes en la zona de estudio, como la humedad del aire y la humedad presente en la cobertura vegetal, la variabilidad del relieve, el viento, la nubosidad y la dispersión y el ángulo de incidencia de la luz solar, los cuales son aspectos a considerar cuando se trabaja con este tipo de información [44]. Igualmente, la información satelital tiene sus limitantes en cuanto a correcciones atmosféricas, geográficas, resolución espacial y nubosidad. 


\section{CONCLUSIONES Y RECOMENDACIONES}

La presente investigación permite sentar las bases del uso de datos multi e hiperespectrales así como la identificación de las diferencias inherentes entre ellas para el análisis de pastos tropicales dedicados a la actividad de pastoreo de ganado lechero con rotación de parcelas.

La información de reflectancia, a partir de información satelital, sobrestima la generada a partir de espectrorradiometría de campo. En este caso, se observa un mayor distanciamiento en el rango del infrarrojo cercano.

Se determinó que el pastoreo del ganado lechero promueve la heterogeneidad en la cobertura del pasto Estrella Africana, influyendo directamente sobre el índice de área foliar y la información hiperespectral del cultivo. Dichas variaciones también están asociadas a la diferencia temporal del desarrollo del cultivo a lo interno de las áreas de pastoreo estudiadas y a su período de recuperación entre ciclos de pastoreo.

La información satelital del IAF generada por la herramienta SNAP sobrestima los valores observados en campo, identificándose así la limitante de estimar, mediante información multiespectral satelital, la condición de campo del IAF para pastos con pastoreo de ganado lechero con rotación de parcelas.

Debido a que las condiciones climáticas varían dependiendo de la hora de recolección de los datos así como de las variaciones de iluminación presentes en la zona, tal y como se menciona en [44] en conjunto con el error de geolocalización, al comparar los índices espectrales de vegetación generados a partir de información satelital e hiperspectral de campo se obtuvieron diferencias significativas.

Es necesario relacionar la firma hiperespectral obtenida en campo con la etapa fenológica del pasto Estrella Africana y las características nutricionales del mismo con el fin de analizar de una manera más integral el estado del cultivo y ofrecer una herramienta más robusta para la toma de decisiones asociadas al cuidado y mantenimiento de este cultivo.

Se recomienda realizar este tipo de estudios durante un período más amplio de tiempo, de manera que se contemple el comportamiento de crecimiento fenológico de los pastos a lo largo del año y así permitir una mayor recolección de datos satelitales sin la influencia de la nubosidad y llevar a cabo un análisis comparativo del efecto de la estacionalidad sobre la información espectral. Además, es necesario contemplar un bloque de control para analizar el efecto del pastoreo respecto a una zona donde el cultivo se desarrolle sin ningún disturbio.

\section{AGRADECIMIENTOS}

Se agradece al Dr. Jorge Alberto Elizondo Salazar, director de la Estación Experimental de Ganado Lechero Alfredo Volio Mata por permitir el uso de las parcelas con pasto Estrella Africana para llevar a cabo esta investigación; al Dr. Werner Rodríguez Montero por proporcionar el analizador de dosel y al proyecto $\mathrm{N}^{\circ} 340-\mathrm{B} 5-507$ inscrito en la Vicerrectoría de Investigación por el financiamiento otorgado para el desarrollo de la investigación. 


\section{ROLES DE AUTORES}

Alberto Francisco Coto Fonseca: conceptualización, investigación, metodología, análisis formal, visualización, redacción (borrador inicial), redacción (revisión y edición)

Alejandra María Rojas González: conceptualización, investigación, metodología, adquisición de fondos, administración del proyecto, recursos, supervisión, redacción (borrador inicial), redacción (revisión y edición)

\section{REFERENCIAS}

[1] Z. Chen et al., "Monitoring and Management of Agriculture with Remote Sensing", en Advances in Land Remote Sensing, Dordrecht: Springer Netherlands, 2008, pp. 397-421.

[2] J. Wang et al., "Estimating leaf area index and aboveground biomass of grazing pastures using Sentinel-1, Sentinel-2 and Landsat images", ISPRS J. Photogramm. Remote Sens., vol. 154, pp. 189-201, ago. 2019, doi: 10.1016/j.isprsjprs.2019.06.007.

[3] J. G. P. W. Clevers, L. Kooistra, y M. E. Schaepman, "Estimating canopy water content using hyperspectral remote sensing data", Int. J. Appl. Earth Obs. Geoinf., vol. 12, núm. 2, pp. 119-125, abr. 2010, doi: 10.1016/j.jag.2010.01.007.

[4] R. G. Allen, A. Kilic, R. Trezza, y J. M. H. Hendrickx, "Satellite-based ET estimation in agriculture using SEBAL and METRIC", Hydrol. Process., vol. 25, núm. 26, pp. 4011-4027, 2011, doi: 10.1002/ hyp.8408.

[5] R. G. Allen et al., "Satellite-Based Energy Balance for Mapping Evapotranspiration with Internalized Calibration METRIC-Applications", J. Irrig. Drain. Eng., vol. 4, núm. 133, pp. 395-406, 2007, doi: 10.1061/(ASCE)0733-9437(2007)133.

[6] L. Mateos y C. Alameda, "Spectral vegetation indices for benchmarking water productivity of irrigated cotton and sugarbeet crops", Agric. Water Manag., vol. 95, pp. 48-58, 2008, doi: 10.1016/j. agwat.2007.09.001.

[7] S. Sovoe, "Mapping Irrigated Area Fragments for Crop Water Use Assessment Using Handheld Spectroradiometer", Int. J. Agron., p. 8, 2011, doi: https://doi.org/10.1155/2011/974040.

[8] A. Mokhtari, H. Noory, M. Vazifedoust, y M. Bahrami, "Estimating net irrigation requirement of winter wheat using model- and satellite-based single and basal crop coe ffi cients", Agric. Water Manag., vol. 208, pp. 95-106, 2018, doi: 10.1016/j.agwat.2018.06.013.

[9] A. Mokhtari et al., "Calculating potential evapotranspiration and single crop coefficient based on energy balance equation using Landsat 8 and Sentinel-2", ISPRS J. Photogramm. Remote Sens., vol. 154, pp. 231-245, 2019, doi: 10.1016/j.isprsjprs.2019.06.011.

[10] M. Drusch et al., "Sentinel-2: ESA's Optical High-Resolution Mission for GMES Operational Services”, Remote Sens. Environ., vol. 120, pp. 25-36, may 2012, doi: 10.1016/j.rse.2011.11.026.

[11] T. R. Loveland y J. R. Irons, "Landsat 8: The plans, the reality, and the legacy", Remote Sens. Environ., vol. 185, pp. 1-6, nov. 2016, doi: 10.1016/j.rse.2016.07.033.

[12] Q. Xie et al., "Retrieval of crop biophysical parameters from Sentinel-2 remote sensing imagery", Int. J. Appl. Earth Obs. Geoinf., vol. 80, pp. 187-195, ago. 2019, doi: 10.1016/j.jag.2019.04.019. 
[13] M. Guerini Filho, T. M. Kuplich, y F. L. F. De Quadros, "Estimating natural grassland biomass by vegetation indices using Sentinel 2 remote sensing data", Int. J. Remote Sens., vol. 41, núm. 8, pp. 2861-2876, abr. 2020, doi: 10.1080/01431161.2019.1697004.

[14] E. Ben-dor et al., "Remote Sensing of Environment Using Imaging Spectroscopy to study soil properties”, Remote Sens. Environ., vol. 113, pp. S38-S55, 2009, doi: 10.1016/j.rse.2008.09.019.

[15] T. Jarmer, M. Vohland, H. Lilienthal, y E. Schnug, "Estimation of Some Chemical Properties of an Agricultural Soil by Spectroradiometric Measurements", Pedosph. An Int. J., vol. 18, núm. 2, pp. 163-170, 2008, doi: https://doi.org/10.1016/S1002-0160(08)60004-1.

[16] I. Melendez-Pastor, J. Navarro-pedreño, I. Gómez, y M. Koch, "Identifying optimal spectral bands to assess soil properties with VNIR radiometry in semi-arid soils", Geoderma, vol. 147, pp. 126-132, 2008, doi: 10.1016/j.geoderma.2008.08.004.

[17] R. E. N. Hong-yan, Z. Da-fang, A. N. Singh, P. A. N. Jian-jun, y Q. I. U. Dong-sheng, "Estimation of As and $\mathrm{Cu}$ Contamination in Agricultural Soils Around a Mining Area by Reflectance Spectroscopy : A Case Study", Pedosph. An Int. J., vol. 19, núm. 6, pp. 719-726, 2009, doi: 10.1016/S10020160(09)60167-3.

[18] N. Bao, L. Wu, K. Yang, y W. Zhou, “Assessing soil organic matter of reclaimed soil from a large surface coal mine using a field spectroradiometer in laboratory", Geoderma, núm. 288, pp. 47-55, 2017, doi: https://doi.org/10.1016/j.geoderma.2016.10.033.

[19] S. N. Kulkarni y R. R. Deshmukh, "Monitoring Carbon, Nitrogen, Phosphor and Water Contents of Agricultural Soil by Reflectance Spectroscopy using ASD Fieldspec Spectroradiometer", Int. J. Eng. Sci. Comput., vol. 6, núm. 11, pp. 3429-3433, 2016.

[20] I. F. S. Gazala et al., "Spectral reflectance pattern in soybean for assessing yellow mosaic disease", Indian J. Virol., vol. 24, núm. 2, pp. 242-249, sep. 2013, doi: 10.1007/s13337-013-0161-0.

[21] M. L. Gnyp et al., "Hyperspectral Analysis of Rice Phenological Stages on Northeast China", ISPRS Ann. Photogramm. Remote Sens. Spat. Inf. Sci., vol. I-7, pp. 77-82, jul. 2012, doi: 10.5194/isprsannals-I-7-77-2012.

[22] R. R. Pullanagari, I. J. Yule, M. P. Tuohy, M. J. Hedley, R. A. Dynes, y W. M. King, "In-field hyperspectral proximal sensing for estimating quality parameters of mixed pasture", Precis. Agric., vol. 13, núm. 3, pp. 351-369, jun. 2012, doi: 10.1007/s11119-011-9251-4.

[23] J. Elizondo Salazar, "Pasto Estrella Africana: Características Nutricionales y Aspectos de Manejo", Vent. Leche., vol. 3, núm. 10, pp. 26-27, 2008.

[24] CIA, IICA, INTA, y Asociacion Costarricense de la Ciencia del Suelo, "Geoportal de Suelos de Costa Rica”, Suelos.cr. http://www.suelos.cr (accesado en Ene. 25, 2020).

[25] R. McCoy M, "Sampling in the Field", en Field Methods in Remote Sensing, New York, NY: The Guilford Press, 2005, pp. 12-40.

[26] A. F. . Goetz, "Making Accurate Field Spectral Reflectance Measurements", Malvern Panalytical. https://www.materials-talks.com/wp-content/uploads/2018/01/Making-Accurate-Field-Spectral-Reflectance-Measurements-LR.pdf (accesado en Mar. 06, 2021).

[27] I. Herrmann, A. Pimstein, A. Karnieli, Y. Cohen, V. Alchanatis, y D. J. Bonfil, "LAI assessment of wheat and potato crops by VEN $\mu$ S and Sentinel-2 bands", Remote Sens. Environ., vol. 115, núm. 8, pp. 2141-2151, ago. 2011, doi: 10.1016/j.rse.2011.04.018. 
[28] W. William et al., "Searching for the Optimal Sampling Desing for Measuring LAI in a Upland Forest", CEUR Workshop Proceedings. http://ceur-ws.org/Vol-1328/GSR2_Woodgate.pdf (accesado en Sep. 14, 2019).

[29] ASD Inc, "ViewSpec Pro Software Install", Malvern Panalytical. https:/www.malvernpanalytical. com/en/support/product-support/software/ViewSpecProSoftwareInstall (accesado en Oct. 01, 2019).

[30] ESA, "Sentinel-2 Spectral Response Functions (S2-SRF)", Sentinel 2 Document Library. https:// earth.esa.int/web/sentinel/user-guides/sentinel-2-msi/document-library/-/asset_publisher/Wk0TKajiISaR/content/sentinel-2a-spectral-responses (accesado en Nov. 01, 2019).

[31] QGIS Development Team, "QGIS A Free and Open Source Geographic Information System”, QGIS. org. http://qgis.org (accesado en Sep. 01, 2019).

[32] J. . Rouse, R. . Haas, J. . Schell, y D. . Deering, "Monitoring vegetation systems in the great plains with ERTS", NTRS - NASA Technical Reports Server. https://ntrs.nasa.gov/archive/nasa/casi.ntrs. nasa.gov/19740022614.pdf (accesado en Ene. 25, 2020).

[33] R. G. Allen, M. Tasumi, y R. Trezza, "Satellite-Based Energy Balance for Mapping Evapotranspiration with Internalized Calibration (METRIC)-Model”, J. Irrig. Drain. Eng., vol. 4, núm. 133, pp. 380-394, 2007.

[34] M. Tasumi, "Progress in operational estimation of regional evapotranspiration using satellite imagery", University of Idaho, 2003.

[35] Ø. Hammer, D. A. . Harper, y R. P.D, "PAST: Paleontological statistics software package for education and data analysis", Palaeontologia Electronica. https://palaeo-electronica.org/2001_1/past/past. pdf (accesado en Mar. 01, 2020).

[36] A. K. Mahlein, U. Steiner, H. W. Dehne, y E. C. Oerke, "Spectral signatures of sugar beet leaves for the detection and differentiation of diseases", Precis. Agric., vol. 11, núm. 4, pp. 413-431, 2010, doi: 10.1007/s11119-010-9180-7.

[37] K. Yu et al., "Hyperspectral Canopy Sensing of Wheat Septoria Tritici Blotch Disease", Front. Plant Sci., vol. 9, ago. 2018, doi: 10.3389/fpls.2018.01195.

[38] H. Croft, J. Arabian, J. M. Chen, J. Shang, y J. Liu, "Mapping within-field leaf chlorophyll content in agricultural crops for nitrogen management using Landsat-8 imagery", Precis. Agric., vol. 21, núm. 4, pp. 856-880, ago. 2020, doi: 10.1007/s11119-019-09698-y.

[39] ASD, Analytical Spectral Devices, Inc. (ASD) Technical Guide. CO, USA, 1999.

[40] T. . Pande, I. Valentine, K. Betteridge, A. Mackay, y D. Horne, "Pasture damage and regrowth from cattle treading", Proc. New Zeal. Grassl. Assoc., núm. 62, pp. 155-160, ene. 2000, doi: 10.33584/ jnzg.2000.62.2366.

[41] R. WingChing-Jones, G. Cabalceta-Aguilar, y A. Alvarado-Hernández, "Impacto del pastoreo con ganado Holstein y Jersey sobre la densidad aparente de un andisol", Agron. Mesoam., vol. 20, núm. 2, p. 371, feb. 2009, doi: 10.15517/am.v20i2.4953.

[42] M. Kganyago, P. Mhangara, T. Alexandridis, G. Laneve, G. Ovakoglou, y N. Mashiyi, "Validation of sentinel-2 leaf area index (LAI) product derived from SNAP toolbox and its comparison with global LAI products in an African semi-arid agricultural landscape", Remote Sens. Lett., vol. 11, núm. 10, pp. 883-892, oct. 2020, doi: 10.1080/2150704X.2020.1767823. 
[43] M. Rossi, G. Niedrist, S. Asam, G. Tonon, E. Tomelleri, y M. Zebisch, "A Comparison of the Signal from Diverse Optical Sensors for Monitoring Alpine Grassland Dynamics”, Remote Sens., vol. 11, núm. 3, p. 296, feb. 2019, doi: 10.3390/rs11030296.

[44] R. Martínez-Barbáchano y G. Solís-Miranda, "Caracterización Espectral y Detección de Flecha Seca en Palma Africana en Puntarenas, Costa Rica”, Rev. Geográfica América Cent., vol. 2, núm. 61, p. 329, jul. 2018, doi: 10.15359/rgac.61-2.13. 